\title{
CMOS IMAGER BASED ON SINGLE PHOTON AVALANCHE DIODES
}

\author{
Cristiano Niclass, Alexis Rochas, Pierre-André Besse, Radivoje S. Popovic, and Edoardo Charbon \\ Ecole Polytechnique Fédérale de Lausanne (EPFL) \\ Switzerland
}

\begin{abstract}
In this paper we report on a $32 \times 32$ optical imager based on single photon avalanche diodes integrated in CMOS technology. The maximum measured dynamic range is $120 \mathrm{~dB}$ and the minimum noise equivalent intensity is $1.3 \times 10^{-3} \mathrm{~lx}$. The minimum integration time per pixel is $4 \mu \mathrm{s}$. The output of each pixel is digital, thereby requiring no complex read-out circuitry, no amplification, no sample \& hold, and no ADC.
\end{abstract}

Keywords: CMOS avalanche photodiodes, SPAD arrays, single photon detectors, high speed imagers.

\section{INTRODUCTION}

Industrial and scientific applications involving the observation and measurement of fast phenomena are enabled by high speed imagers. High speed is related to ability of providing either large numbers of frames per second or lower frame rates with ultra-short and welldefined integration times. Analysis of neuronal activity in vivo or in vitro using voltage-sensitive dyes (VSD) [1], fluidic flow measurements based on particle image velocimetry (PIV) [2], and fluorescence lifetime imaging using gating techniques [3] are examples of such applications.

For instance, the analysis of neuronal activity requires the measurement of electrical potentials in the tissue with simultaneously high spatial (few tens of $\mu \mathrm{m}$ ) and temporal resolutions (few ms). These requirements make solid-state imagers combined with VSD a much more suitable technique if compared to the use of electrodes inserted into the tissue [1]. So far, the image sensor of choice has been CCD. High performance CCD imaging systems are however costly and consequently prohibitive for a number of new applications. With the recent introduction of true photon counting detectors integrated in CMOS technology [4], highly sensitive miniaturized cameras are now feasible.

In this paper we propose a new grayscale image sensor based on single photon avalanche diodes integrated in CMOS technology. Its potential for high sensitivity and speed are evaluated here. The main performance parameters for the proposed imager are described and corroborated by measurement results.

\section{SINGLE PHOTON AVALANCHE DIODES}

An avalanche photodiode reverse-biased above its breakdown voltage $\left(V_{b d}\right)$ allows single photon detection [4]. When such a diode is biased above $V_{b d}$, it remains in a zero current state for a relatively long period of time, usually in the millisecond range. During this time, a very high electric field exists within the $p-n$ junction forming the avalanche multiplication region. Under these conditions, if a primary carrier enters the multiplication region and triggers an avalanche process, several hundreds of thousands of secondary electron-hole pairs are generated by impact ionization, thus causing the diode's depletion capacitance to be rapidly discharged. As a result, a sharp current pulse is generated and can be easily measured. This mode of operation is commonly known as Geiger mode.

Unfortunately, typical photodiodes, as those used in conventional imagers, are not compatible with this mode of operation since they suffer from a premature breakdown when the bias voltage approaches $V_{b d}$. The peak electric field is located only in the diode's periphery rather than in the planar region, which causes premature breakdown [4]. A single photon avalanche diode (SPAD), on the other hand, is a specifically designed photodiode in which premature breakdown is avoided and a planar multiplication region is formed within the whole junction area.

Linear mode avalanche photodiodes, which are biased just below $V_{b d}$, have a finite multiplication gain. Statistical variations of this finite gain produce an additional noise contribution known as excess noise. SPADs, on the other hand, are not concerned with these gain fluctuations since the optical gain is virtually infinite. Nevertheless, the statistical nature of the avalanche buildup is translated onto a detection probability. Indeed, the probability of detecting a photon hitting the SPAD's surface, known as the photon detection probability (PDP), depends on the diode's quantum efficiency and the probability for an electron or for a hole to trigger an avalanche. Additionally, in Geiger mode, the signal amplitude does not provide intensity information since all the current pulses have the same amplitude. Intensity information is however obtained by counting the pulses during a certain period of time or by measuring the mean time interval between successive pulses. The same mechanism may be used to evaluate noise. Thermally or tunneling generated carriers within the $\mathrm{p}-\mathrm{n}$ junction, which produce dark current in 
linear mode photodiodes, can trigger avalanche pulses. In Geiger mode, they are indistinguishable from regular photon-triggered pulses and they produce spurious pulses at a frequency known as dark count rate (DCR). DCR strongly depends on temperature and it is an important parameter for imagers since it defines the minimal detectable signal, thus also limiting the dynamic range of the imager.

Another source of spurious counts is represented by after-pulses. They are due to carriers temporarily trapped after a Geiger pulse in the multiplication region that are released after a short time interval, thus re-triggering a Geiger event. After-pulses depend on the trap concentration as well as on the number of carriers generated during a Geiger pulse. The number of carriers depends in turn on the diode's parasitic capacitance and on the external circuit, which is usually the circuit used to quench the avalanche. Typically, the quenching process is achieved by temporarily lowering the bias voltage below $\mathrm{V}_{\mathrm{bd}}$. Once the avalanche has been quenched, the SPAD needs to be recharged again above $V_{b d}$ so that it can detect subsequent photons. The time required to quench the avalanche and recharge the diode up to $90 \%$ of its nominal excess bias is defined as the dead time. This parameter limits the maximal rate of detected photons, thus producing a saturation effect. The dead time consequently limits the dynamic range of the image sensor.

A useful property of SPADs is the ability of accurately detecting the arrival time of photons. The statistical fluctuation of the time interval between the arrival of a photon at the sensor and the output pulse leading edge is defined as the timing jitter or timing resolution. Timing jitter mainly depends on the time a photo-generated carrier requires to be swept out of the absorption point into the multiplication region.

During an avalanche, some photons can be emitted due to the electroluminescence effect [5]. These photons may be detected by neighboring pixels in an array of SPADs. The probability of this effect is called optical crosstalk probability. This probability is much smaller in fully integrated arrays of SPADs in comparison to hybrid versions. This is due to the fact that the diode's parasitic capacitance in the integrated version is orders of magnitude smaller than the hybrid solutions, thus reducing the energy dissipated during a Geiger event. Electrical crosstalk on the other hand is produced by the fact that photons absorbed beyond the $\mathrm{p}-\mathrm{n}$ junction, deep in the substrate, generate carriers which can diffuse to neighboring pixels. The probability of occurrence of this effect, whether it is optical or electrical, defines the crosstalk probability.

\section{IMAGE SENSOR IMPLEMENTATION}

The imager pixel consists of a circular SPAD and a 5transistor (5T) configuration. The cross-section of the SPAD is shown in Fig. 1. The device was fabricated in a high voltage $0.8 \mu \mathrm{m}$ CMOS process. It is a dual $\mathrm{p}+/$ deep n-tub/p-substrate junction. The planar $\mathrm{p}+/$ deep $\mathrm{n}$-tub junction provides the multiplication region where the Geiger breakdown occurs. The fabrication process was a $2 \mathrm{M} / 2 \mathrm{P}$ twin-tub technology on a p-substrate allowing an operating voltage of 2.5 to $50 \mathrm{~V}$. Premature breakdown is avoided by surrounding the photodiode with a guard ring of relatively lightly doped diffusion. The guard ring eliminates abrupt doping profiles and corner effects by reducing electric field gradients at the diode's periphery. A useful feature of this technology is the availability of a p-tub implantation to create such a ring surrounding the $\mathrm{p}+$ region anode [4]. The breakdown voltage $V_{b d}$ of the $\mathrm{p}+/$ deep $\mathrm{n}$-tub junction is $25.5 \mathrm{~V}$. A larger bias voltage $V_{b d}+V_{e}$ is applied on the diode to operate in single photon detection mode. $V_{e}$ is known as excess bias voltage.

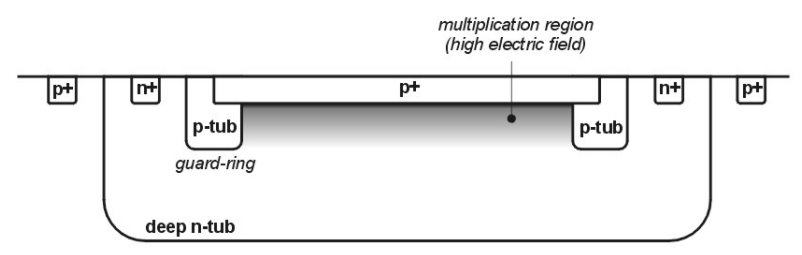

Fig. 1. SPAD cross-section.

Fig. 2 shows the pixel circuit and a photomicrograph of the structure. The SPAD $\mathrm{p}+$ anode is nominally biased at a negative voltage $V_{o p}$ equal to $-25.5 \mathrm{~V}$. This voltage is common to all the pixels in the array. The deep n-tub cathode is connected to the power supply $V D D=5 \mathrm{~V}$ through a long channel p-mos transistor $T_{q}$. The excess bias voltage $V_{e}$ is thus equal to $\left|V_{o p}\right|+V D D-V_{b d}=5 \mathrm{~V}$.
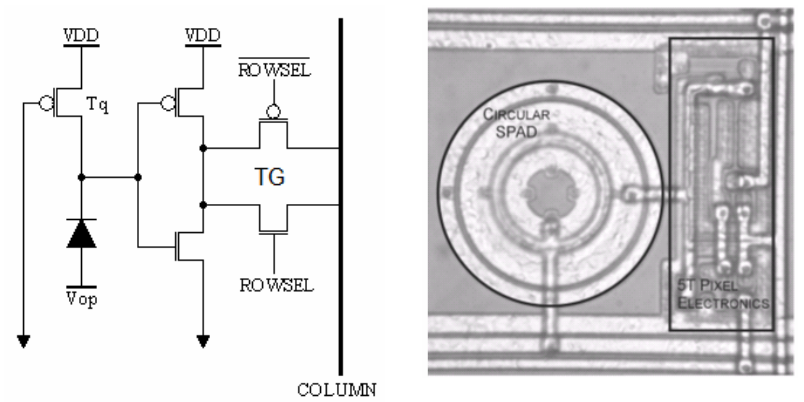

Fig. 2. 5T pixel circuit and pixel photomicrograph.

The SPAD operates in passive quenching. Upon photon arrival, the breakdown current discharges its depletion region capacitance, causing the bias voltage to drop below breakdown voltage. The $\mathrm{W} / \mathrm{L}$ ratio of $T_{q}$ is set to provide a sufficiently resistive path to quench the avalanche process. After avalanche quenching, the SPAD recharges through $T_{q}$ and progressively recovers its photon detection capability. The time required for quenching the avalanche and restoring the operating bias, i.e. the dead time, is typically less than $40 \mathrm{~ns}$ for the $5 \mathrm{~T}$ digital pixel. 
At the cathode, an inverted analog voltage pulse of amplitude $V_{e}$ reflects the detection of a single photon. The CMOS inverter stage converts this analog voltage pulse into a digital pulse. A transmission gate (TG) is used to feed the detection signal to the nearest column output line when $\mathrm{s}=V D D$ and $\bar{s}=G N D$ (read phase). Every pixel generates a digital pulse which captures the arrival of a photon with picosecond precision. The nearinfinite internal gain inherent to Geiger mode operation leads to no further amplification and the pixel output can be, if needed, routed directly outside the chip. To the best of our knowledge, we are reporting the first scalable and intrinsically digital pixel implemented in CMOS process for grayscale imaging applications. The 5T digital pixel occupies an area of $58 \mu \mathrm{mx} 58 \mu \mathrm{m}$. The active area of the SPAD is $38 \mu \mathrm{m}^{2}$.

The functional diagram of the sensor is shown in Fig. 3 . The sensor consists of an array of $32 \times 32$ pixels and requires two power supply buses $V D D=5 \mathrm{~V}$ and $V_{o p}=-$ $25.5 \mathrm{~V}$. Digital pixels allow the readout to be designed with less stringent constraints on noise performance. Moreover, no amplification, no sample \& hold, and no A/D converter are necessary. Consequently, in SPAD based image sensors, performance is not limited by analog noise and device mismatch in the readout circuitry. Therefore no particular care has to be given to the design of those components, except for minimizing digital noise and optimizing speed. In this implementation, the readout circuitry consists of a 5-bit decoder for row selection and a 32-to-1 multiplexer (MUX) for column selection.

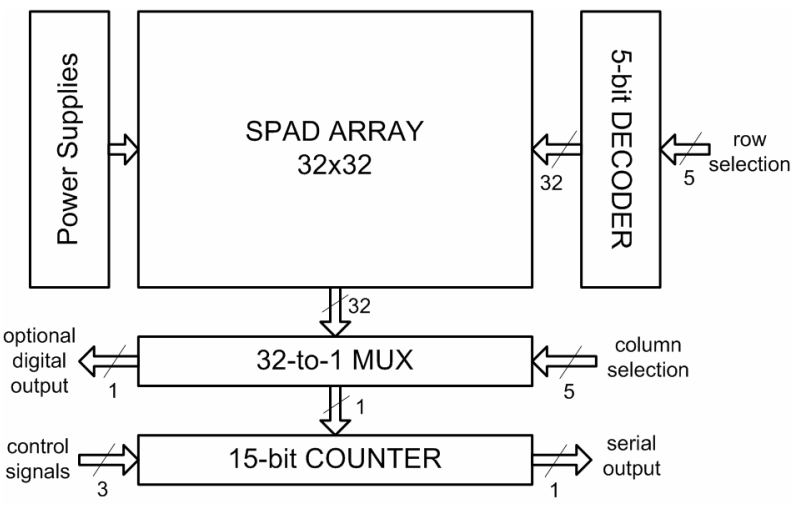

Fig. 3. Functional diagram of the image sensor.

A fast 15-bit linear feedback shift register counter has been implemented on-chip and it is used to compute intensity images. Intensity information is obtained by counting detection pulses within the integration time. Addressing and control signals were provided using a National Instruments PXI acquisition rack. The MUX output is also externally used for testing and characterization purposes.

\section{MEASUREMENT RESULTS}

Fig. 4 shows the measured PDP as a function of the photon wavelength for a typical pixel with a nominal $V_{e}$ of $5 \mathrm{~V}$ at room temperature. It is larger than $20 \%$ between $430 \mathrm{~nm}$ and $570 \mathrm{~nm}$ with a peak at $26 \%$ at $460 \mathrm{~nm}$. At $700 \mathrm{~nm}$ the PDP is still $8 \%$ without any post process treatment of the silicon chip surface.

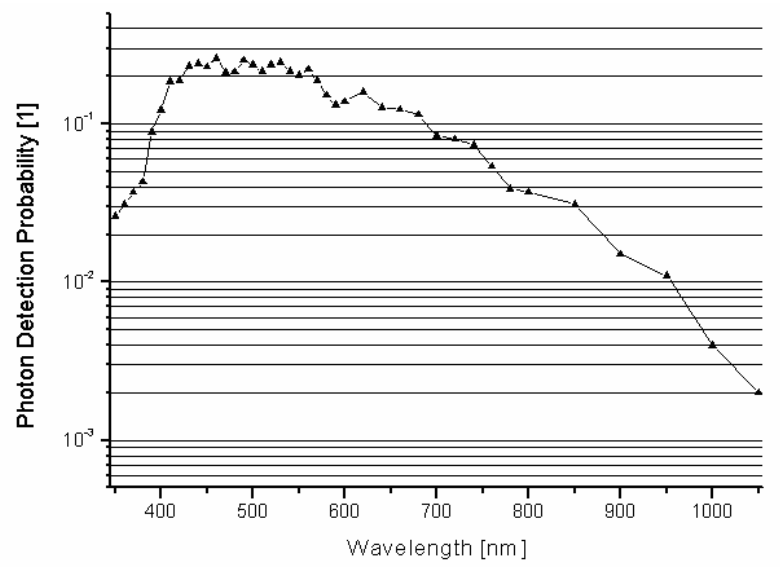

Fig. 4. Photon detection probability.

The distribution of DCR across the sensor array for nominal $V_{e}$ is plotted in Fig. 5. At room temperature, the limited active area of the SPAD and the outstanding cleanliness of the CMOS process lead to a DCR of $350 \mathrm{~Hz}$ on average in the array and negligible afterpulsing effects. In Fig. 6, the distribution of DCR is plotted for $\mathrm{T}=0^{\circ} \mathrm{C}$. In this case, the mean value of the DCR dropped below $75 \mathrm{~Hz}$. Fig. 7 shows a histogram of the DCR distribution. Note that $95 \%$ of the SPADs in the array exhibit a DCR below $1 \mathrm{kHz}$ at room temperature. At $\mathrm{T}=0^{\circ} \mathrm{C}, 61 \%$ of the SPADs exhibit an outstanding DCR level below $1 \mathrm{~Hz}$. In addition, almost $93 \%$ of them showed a DCR below $100 \mathrm{~Hz}$.

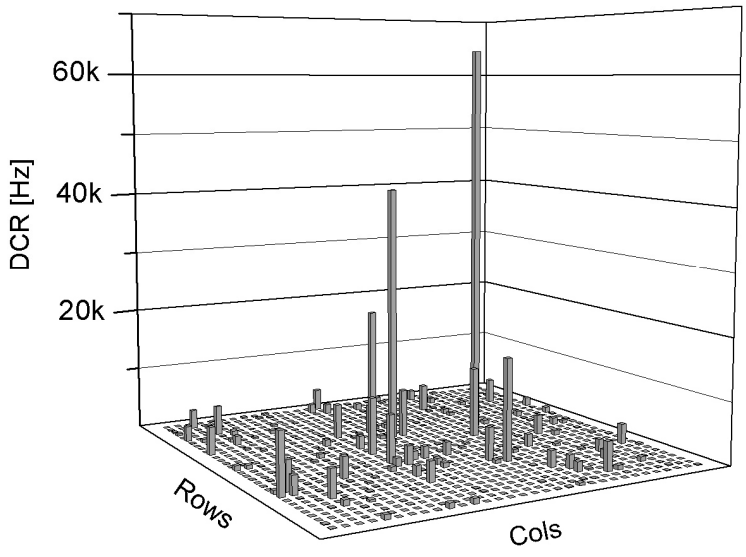

Fig. 5. Dark count rate at room temperature. 


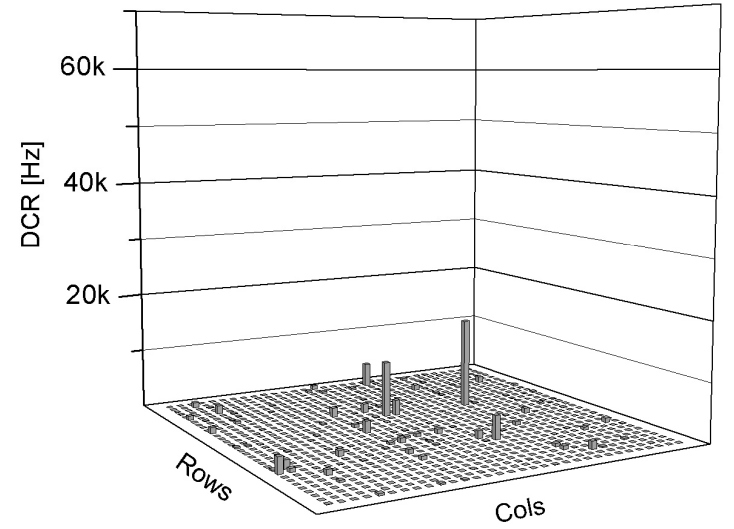

Fig. 6. Dark count rate at $\mathrm{T}=0^{\circ} \mathrm{C}$.

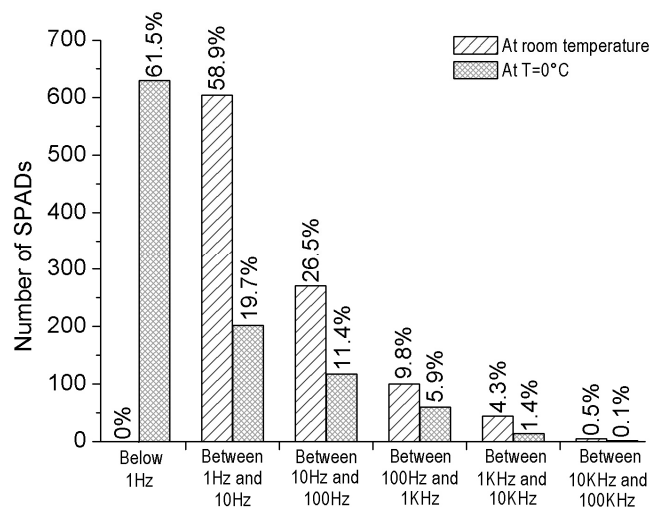

Fig. 7. Histogram of DCR at room temperature and $\mathrm{T}=0^{\circ} \mathrm{C}$.

Fig. 8 shows the timing jitter measurement of the pixel. The measurement setup consisted of a pulsed laser source with pulse width of $30 \mathrm{ps}$ used to excite a single pixel through an optical fiber. An oscilloscope with 2ps of uncertainty was used to measure the time interval between the laser output trigger and the sensor output signal. The resulting overall timing jitter was $115 \mathrm{ps}$ FWHM.

Experimental measurements demonstrated that the SPAD array is free of crosstalk effects. Crosstalk was measured by illuminating a single pixel in the center of the array using a highly focused laser beam through the optics of a microscope. Optical crosstalk was expected to be negligible thanks to very small parasitic capacitance. Electrical crosstalk was eliminated by design. Minor carriers diffusing in the substrate cannot reach the multiplication region of a pixel since they would be collected by the deep n-tub/p-substrate junction.

Fig. 9 illustrates the optical response of a typical pixel to a variable illumination measured with an integration time of $1 \mathrm{~s}$. The sensor was tested with a homogenous illumination using a monochromator whose wavelength was set to $635 \mathrm{~nm}$. At a high intensity illumination, when the signal approached 20 mega counts, the SPAD presented a saturation behavior due to the dead time [6].
This point is considered to be the saturation level. Due to the absence of readout noise, when the illumination is very low, the signal is only limited by the noise in the dark. Since dark counts respect a Poissonian distribution [4], their average value can be electronically stored for each pixel and removed from the final image. Under this assumption, the temporal noise in the dark is defined as the time varying component of the dark counts, i.e. the square root of them. At room temperature, the average temporal noise in the dark was 18 counts. The noise equivalent intensity is defined as the optical intensity where the SNR is equal to $0 \mathrm{~dB}$ and designates the minimum detectable signal. It was $3.2 \times 10^{-10} \mathrm{~W} / \mathrm{cm}^{2}$ for the proposed imager under these conditions, which corresponds to $1.3 \times 10^{-3} \mathrm{~lx}$. As a result, a dynamic range (DR) of $120 \mathrm{~dB}$ was obtained.

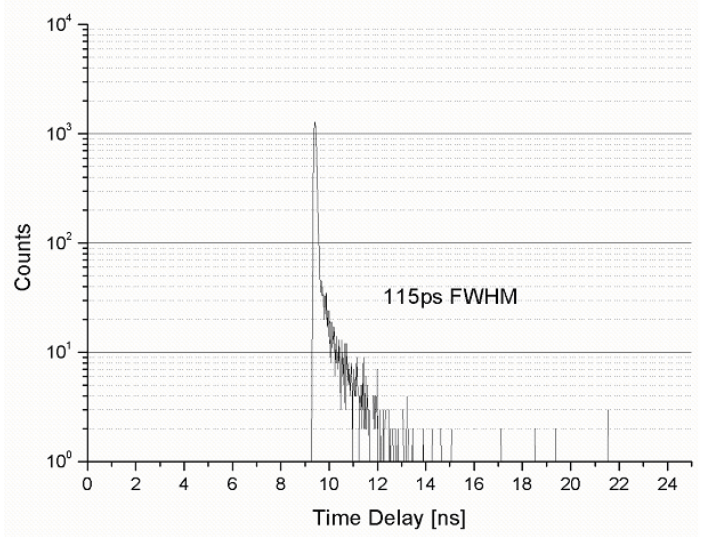

Fig. 8. Histogram of the measured timing jitter.

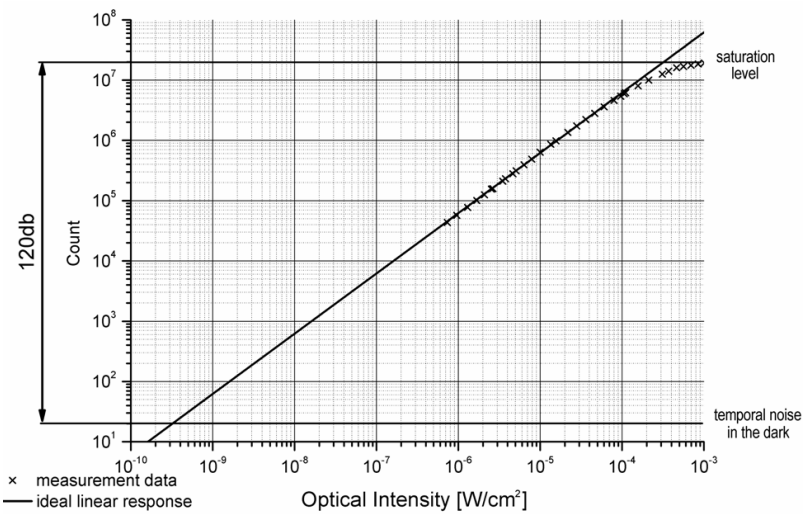

Fig. 9. Pixel optical response and dynamic range.
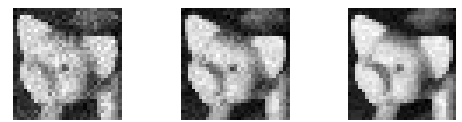

$4 \mu \mathrm{s}$

$10 \mu \mathrm{s}$

$25 \mu \mathrm{s}$
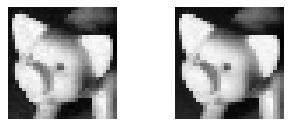

$100 \mu \mathrm{s}$

$1 \mathrm{~ms}$

Fig. 10. Image of a model at different integration times. 
The SPAD based image sensor was tested in real time setup coupled to a camera objective at room temperature. Fig. 10 shows examples of images taken at various integration times down to $4 \mu \mathrm{s}$. The scene was illuminated using a 50W halogen light. Table 1 summarizes the salient performance measurements of the imager.

\section{CONCLUSION}

In this paper we reported the design and characterization of a grayscale CMOS image sensor based on an array of $32 \times 32$ SPADs. This type of imager differs considerably from conventional CMOS active pixel sensors. The performance parameters have been described and experimentally measured. Due to the outstanding noise performance of the proposed imager, a noise equivalent intensity of $1.3 \times 10^{-3} \mathrm{~lx}$ and a pixel dynamic range of $120 \mathrm{~dB}$ were obtained. Images of acceptable quality have been recorded with integration times in the microsecond range. These parameters make SPAD based image sensors very suitable for applications requiring simultaneously high sensitivity and very short integration times. Parallel readout circuits, currently been designed, will eventually enable acquisition of multithousand images per second. Finally, due to their superior timing resolution, combined with high sensitivity, SPAD arrays are a low-cost alternative to image intensifiers, when picosecond time discrimination is required [3].

Table 1. Performance summary

\begin{tabular}{|l|l|c|c|c|}
\hline \hline Level & Measurement & Symbol & Value & Unit \\
\hline Pixel & $\begin{array}{l}\text { Photon detection } \\
\text { probability peak @ 460nm }\end{array}$ & PDP & 26 & $\%$ \\
\cline { 2 - 5 } & $\begin{array}{l}\text { Average dark count rate @ } \\
\text { T=27ㄷ }\end{array}$ & DCR & 350 & $\mathrm{~Hz}$ \\
\cline { 2 - 5 } & Fill factor & - & 1.1 & $\%$ \\
\cline { 2 - 5 } & FWHM timing jitter & - & 115 & $\mathrm{ps}$ \\
\cline { 2 - 5 } & Dead time & - & $<40$ & $\mathrm{~ns}$ \\
\cline { 2 - 5 } & Dynamic range & - & 4 & $\mu \mathrm{s}$ \\
\hline Sensor & Minimum integration time & - & $1.3 \cdot 10^{-3}$ & $1 \mathrm{x}$ \\
\cline { 2 - 5 } & $\begin{array}{l}\text { Optical intensity @ } \\
\text { SNR=0dB }\end{array}$ & - & 7 & $\mathrm{~mm}^{2}$ \\
\cline { 2 - 5 } & Chip size & $\mathrm{f} / \#$ & $\mathrm{f} / 3.9$ & - \\
\hline Lens & F-number & & & $\mathrm{dB}$ \\
\hline \hline
\end{tabular}

\section{Acknowledgements}

This research was supported by a grant of the Swiss National Science Foundation - Grant Nr.: 620-066110.

\section{References}

[1] J. Fisher et al., "In vivo fluorescence microscopy of neuronal activity in three dimensions by use of voltage-sensitive dyes", Optics Letters, Vol. 29, No. 1, pp 71-73, Jan. 2004.

[2] H. M. Fritz, "PIV Applied to landslide generated impulse waves", $10^{\text {th }}$ Intl. Symp. on Applications of Laser Techniques to Fluid Mechanics, Jul. 2000.

[3] K. Dowling et al., “Two Dimensional Fluorescence Lifetime Imaging using a $5 \mathrm{kHz} / 100 \mathrm{ps}$ Gated Image Intensifier”, BIOS Meeting, 1997.

[4] A. Rochas, "Single Photon Avalanche Diodes in CMOS technology", Ph.D. Thesis, EPF-Lausanne, 2003.

[5] R. H. Haitz, "Studies on optical coupling between silicon p-n junctions", Solid State Electronics, 8, pp. 417-425, 1965.

[6] C. Niclass et al., "Toward a 3-D Camera Based on Single Photon Avalanche Diodes", IEEE Journal of Selected Topics in Quantum Electronics, Vol. 10, No. 4, pp 796-802, Jul./Aug. 2004. 